\title{
Prediction Method of TBM Tunneling Parameters Based on Bi-GRU-ATT Model
}

\author{
Qinglong Zhang $\mathbb{D}^{1},{ }^{1}$ Boyu Yang, ${ }^{2}$ Yanwen Zhu, ${ }^{1}$ Chen Guo, ${ }^{2}$ Chong Jiao, ${ }^{2}$ and Anmin Cai $\mathbb{i D}^{2}$ \\ ${ }^{1}$ Department of Civil Engineering, School of Civil and Resource Engineering, University of Science and Technology Beijing, \\ Beijing 100083, China \\ ${ }^{2}$ China Huaneng Clean Energy Research Institute, Beijing 102209, China
}

Correspondence should be addressed to Anmin Cai; am_cai@qny.chng.com.cn

Received 17 December 2021; Accepted 27 December 2021; Published 10 February 2022

Academic Editor: Mingfeng Lei

Copyright (c) 2022 Qinglong Zhang et al. This is an open access article distributed under the Creative Commons Attribution License, which permits unrestricted use, distribution, and reproduction in any medium, provided the original work is properly cited.

\begin{abstract}
With tunnel boring machines (TBMs) widely used in tunnel construction, the adaptable adjustment of TBM operating status has become a research focus. Since the prediction of tunnel geological conditions is still challenging before excavating, the prediction of important TBM operating parameters plays an important role in the research on TBM adaptable adjustment. This paper proposes an intelligent prediction method of TBM tunneling parameters based on bidirectional gate recurrent unit incorporating attention mechanism (Bi-GRU-ATT) and selects a complete tunneling cycle to predict the tunneling parameters of the TBM complete tunneling cycle. Relying on the TBM3 bid section of Jilin Water Supply Project, 21 key parameters of the complete tunneling cycle are selected as the input features of the model to realize the prediction of four tunneling parameters in the complete driving cycle section of TBM. Compared with the Bi-GRU, GRU, and Long Short-Term Memory (LSTM) models, it can be seen that the Bi-GRU-ATT model has a goodness of fit for predicting TBM tunneling parameters above 0.92, and the average absolute percentage error is less than $1.8 \%$. The results show that the prediction method of TBM tunneling parameters based on Bi-GRU-ATT model proposed in this paper has stronger learning and prediction capabilities. This prediction method provides a more feasible auxiliary intelligent decision-making method for TBM aided intelligent construction.
\end{abstract}

\section{Introduction}

Tunnel boring machine (TBM) integrating the functions of excavation, support, slag discharge, and transportation is one of the most advanced types of equipment for a variety of tunnel constructions, e.g., traffic, municipal, water conservancy, water supply, and gas transmission pipelines [1]. TBM has gradually replaced the traditional blasting excavation in various tunnel projects due to its high efficiency, safety, and environmental protection [2]. However, the adaptability of TBM is often limited by complex geological conditions, the poor matching of tunneling parameters and rock mass state parameters, and the high requirements on the experience of construction personnel, making it difficult to effectively solve the prediction of TBM tunneling parameters $[3,4]$. Therefore, correct evaluation and prediction of TBM tunneling parameters are important issues in efficient tunnel excavation, which is of great significance for ensuring the safety and efficient construction of TBM.

In recent years, scholars have conducted a lot of research on the prediction of TBM tunneling parameters [5-7] and have achieved certain results. At present, TBM tunneling parameter prediction methods are mainly summarized into empirical formula method (combined with experiment) [8], theoretical analysis method [9], numerical simulation method [10], and machine learning method [11]. In the early empirical method research, Krause [12-14] proposed an empirical model for TBM load prediction, which is of great significance to TBM load calculation. Avunduk and Copur [15] divided the tunnel alignment into three general sections according to geological and geotechnical conditions and proposed an empirical performance prediction model for Earth pressure 
balance (EPB) TBM. Ates et al. [6] recommended empirical models for predicting torque, thrust, and other design parameters. With the continuous improvement of test equipment, multifactor prediction models represented by the Norwegian University of Science and Technology (NTNU) model $[16,17]$ have been widely used. In addition to the above empirical methods, many scholars have also made some research progress in theoretical analysis methods. The Colorado School of Mines (CSM) has developed a well-known theoretical model [18], which analyzes the cutting force estimated through full-scale laboratory cutting tests, so that the maximum PR can be determined by theoretical derivation. Based on this, a number of scholars [19-22] extended the theoretical prediction models. In addition, some scholars predict tunneling performance based on numerical simulation methods. Kasper and Meschke [23] conducted a numerical sensitivity study to analyze the influence of TBM operating parameters and design parameters. Afrasiabi et al. [24] used numerical simulation to study the influence of different factors on TBM tunneling performance. The above studies have proved that the three methods have a certain guiding effect on the prediction of TBM tunneling parameters, but there are still great limitations when facing the complex problems related to TBM tunnels. The empirical formula method can only provide a series of TBM parameter values and cannot sensitively capture the small changes, so its applicability is extremely limited; the theoretical analysis method cannot explain the complex and changeable geology conditions in the various rock types encountered during tunnel construction; the numerical simulation method can only provide the value range of the load parameters, and the calculation is time-consuming, so it cannot provide real-time and effective guidance for the actual construction.

With the development of artificial intelligence technology $[25,26]$, several scholars use machine learning algorithms to analyze complex data problems generated in actual engineering and gradually introduce machine learning algorithms into the research of TBM tunneling parameter prediction. Zhou et al. [27] proposed a support vector machine (SVM) model based on particle swarm optimization (PSO) to predict the energy consumption of shield tunnels. Zhang [28] applied the extreme gradient lifting method to predict the ground settlement caused by the excavation of the EPB shield. In addition. Sun et al. [29] used the random forest to achieve TBM load prediction. Yang et al. [30] utilized support vector regression (SVR) to predict the PR of TBM and took a tunnel in China as an example to study the parameters affecting TBM performance. Wang et al. [31] established a model based on the XGBoost algorithm to implement TBM intelligent decisionmaking. Although machine learning methods can effectively predict TBM tunneling parameters, they cannot consider the time variation characteristics and temporal correlations between TBM tunneling data. For example, the output of SVR or random forest is determined only by the current input without resorting to the network status at the previous moment, and they cannot fully utilize the real-time parameters of TBM tunneling. Therefore, they cannot realize the real-time analysis of TBM tunnels.
To solve this problem, deep learning methods such as recurrent neural networks (RNN) [32] have been developed to model the time series data in the TBM tunnel. Compared with traditional machine learning methods, deep learning is more suitable for learning complex models from high-dimensional data. RNN is a kind of neural network that uses the sequence propagation method to process sequence data. Gao et al. [33] compared and analyzed the effects of different deep learning methods on real-time prediction of TBM tunneling parameters, and the results showed that as an improvement of the RNN network, the recurrent neural network algorithms with gate operations (such as long-short term memory (LSTM) [34] and gate recurrent unit (GRU) [35]) can well solve the problems of gradient explosion and gradient disappearance in RNN networks, and the prediction effect is better than that of the traditional RNN algorithms. However, the GRU network and the LSTM network only consider the association between the sequences in one direction and do not consider the two-way nature of information dissemination, and there are great limitations in dealing with the problem of sequence data with strong correlation before and after. In addition, if the input information is too long, the fixed-length feature vector of the neural network cannot fully store all the context information, which will cause part of the information to be lost or early historical information to be overwritten. To this end, this paper improves its network structure and propagation method and optimizes the model based on the self-attention mechanism, so that the model learns the weight distribution of input information by itself and improves its feature extraction efficiency and learning ability for long-sequence data. Based on this, an intelligent prediction method based on Bi-GRU-ATT integrated with an attention mechanism to solve this problem is proposed.

This paper divides the data set based on the TBM3 bid section of the Jilin Water Supply Project and selects the complete tunneling cycle section data to realize the parameter prediction of the complete tunneling cycle section. Using Pearson correlation analysis, twenty-one parameters with the highest correlation with the predicted parameters are selected as the input characteristics of the model from 199-dimensional tunneling parameters of the complete tunneling cycle section. The data is processed by constructing a binary state discriminant function, eliminating nonworking state and abnormal data, the complete tunneling cycle is extracted, and then the Adam optimizer is used to train the model. The comparative analysis of the BiGRU model, unidirectional LSTM model, and unidirectional GRU model verifies the accuracy and effectiveness of the BiGRU-ATT model in this paper, which has certain guiding significance for TBM intelligent construction.

\section{Recurrent Neural Network}

2.1. Bidirectional Gate Recurrent Unit. Gated Recurrent Unit (GRU), as an improvement of Recurrent Neural Network (RNN), introduces a special gating mechanism to control the retention and forgetting of information, which can effectively deal with the disappearance and/or explosion of 
gradients in variable-length sequences, wait for long-term dependence on the problem, and fall into the problem of local optimal solutions. However, the GRU network can only propagate along the sequence in one direction. When calculating the output of the target vector, the subsequent time information cannot be used. The two-way nature of information propagation is not considered. There is a relatively major limitation in dealing with the sequence data with strong correlation before and after processing.

To solve the above problems, some scholars have improved the propagation mode of the GRU network and proposed a bidirectional GRU network. As shown in Figure 1, the Bi-GRU neural network adds a backpropagation layer to the GRU network structure to control forward and backward output, respectively, thereby improving the accuracy of information classification and realizing two-way processing of context information. The forward propagation layer calculates the sequence information at the current moment from front to back, and the backpropagation layer calculates the same sequence information from back to forward. The two network layers are connected to an output layer. The forward sequence and the reverse sequence are calculated in parallel, and the output result of the target vector is obtained by synthesizing the output of the forward and reverse sequence.

As shown in Figure 1, the structure contains two parallel layers, namely, a forward propagation layer and a backpropagation layer. Each layer is an independent GRU structure. The two structures are symmetrical, and the direction of information transmission is opposite. The input layer information propagates from front to back along the forward sequence, and the reverse sequence is calculated from back to front at the same time. The two are calculated independently and do not interfere with each other. Finally, the output values of the output layer are obtained by synthesizing the output values of the forward and reverse sequence. The calculation equation [36] is as follows:

$$
\begin{aligned}
h_{t}^{\prime} & =f\left(w_{1} x_{t}+w_{3} h_{t}^{\prime}+b_{t}^{\prime}\right), \\
h_{t} & =f\left(w_{2} x_{t}+w_{4} h_{t-1}+b_{t}\right), \\
H_{t} & =h_{t}^{\prime} \oplus h_{t} .
\end{aligned}
$$

The forward sequence and the reverse sequence are calculated by equations (1) and (2), respectively, and finally, the output of the output layer is obtained by equation (3). Among them, $h_{t}^{\prime}$ represents the state of the forward propagation hidden layer at time $t ; h_{t}$ represents the state of the hidden layer backpropagation at time $t ; H_{t}$ represents the output value; $x_{t}$ represents the input value; $\oplus$ represents the vector stitching operation; $w_{1}, w_{2}, w_{3}$, and $w_{4}$ represent the weights to propagate along with the time series.

2.2. Self-Attention Mechanism. In the process of TBM tunneling parameter prediction, the influence of each parameter on the prediction results is different. By analyzing and identifying the importance of each sequence data, the model complexity and prediction performance are optimized. In this paper, the self-attention mechanism layer is introduced to

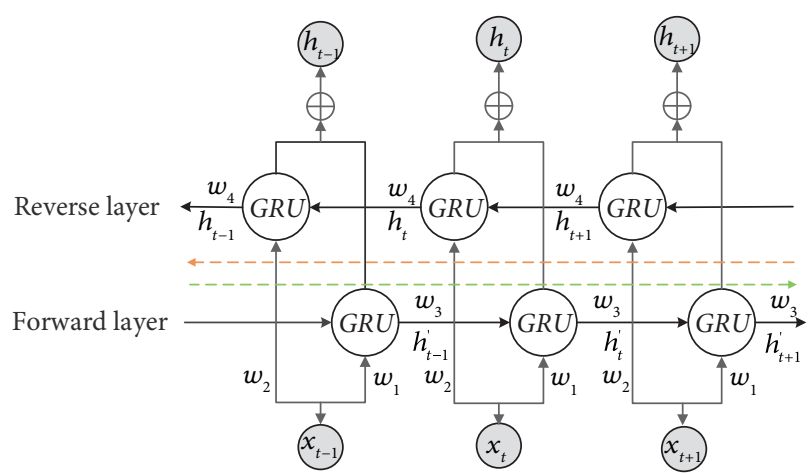

Figure 1: Bi-GRU network structure diagram.

redistribute the weight of the output vector processed by $\mathrm{Bi}$ GRU. Attention mechanism originates from the research in the field of human vision. It can be understood as an encoding-encoding structure in essence. It is manifested in that this mechanism imitates the sequential information processing method of the human brain, which only focuses on key details and ignores unnecessary information when dealing with complex external information. This method can effectively avoid the waste of feature information caused by the direct transmission of the output vector of the Bi-GRU layer to the fully connected layer and indirectly improve the ability of the model to capture key information and improve the prediction efficiency of the model.

The self-attention mechanism is an improvement of the traditional attention mechanism, which reduces the dependence on external information and is better at capturing the internal correlation of data information. The core of this mechanism is the introduction of weight coefficients. By taking the hidden state set of all time steps in the Bi-GRU layer as the model input, the attention vector $c$ is output, which is mainly divided into two calculation processes: weight calculation and weighted summation. The weight calculation equation [37] is as follows:

$$
\begin{aligned}
& e_{t}=\tanh \left(\omega x_{t}+b\right), \\
& a_{t}=\frac{\exp \left(e_{t} A\right)}{\sum_{k=1}^{K} \exp \left(e_{t} A\right)},
\end{aligned}
$$

where $a_{t}$ represents the probability of attention distribution; $x_{t}$ represents the output vector of the Bi-GRU layer; $e_{t} A$ represents the unnormalized attention score. The higher the attention score, the higher the matching degree between the input vector and the target vector; $\omega, A$ and $b$ represent the weight matrix and bias of the attention model, respectively.

According to the attention distribution $a_{t}$, the input vector of the attention layer is weighted and summed to obtain the output vector $\mathrm{c}$ of the attention model [37]:

$$
c=\sum_{k=1}^{K} a_{t} x_{t} .
$$

2.3. Bi-GRU Network with Self-Attention Mechanism. Figure 2 shows the development framework of the hybrid model. The framework module includes configuration 


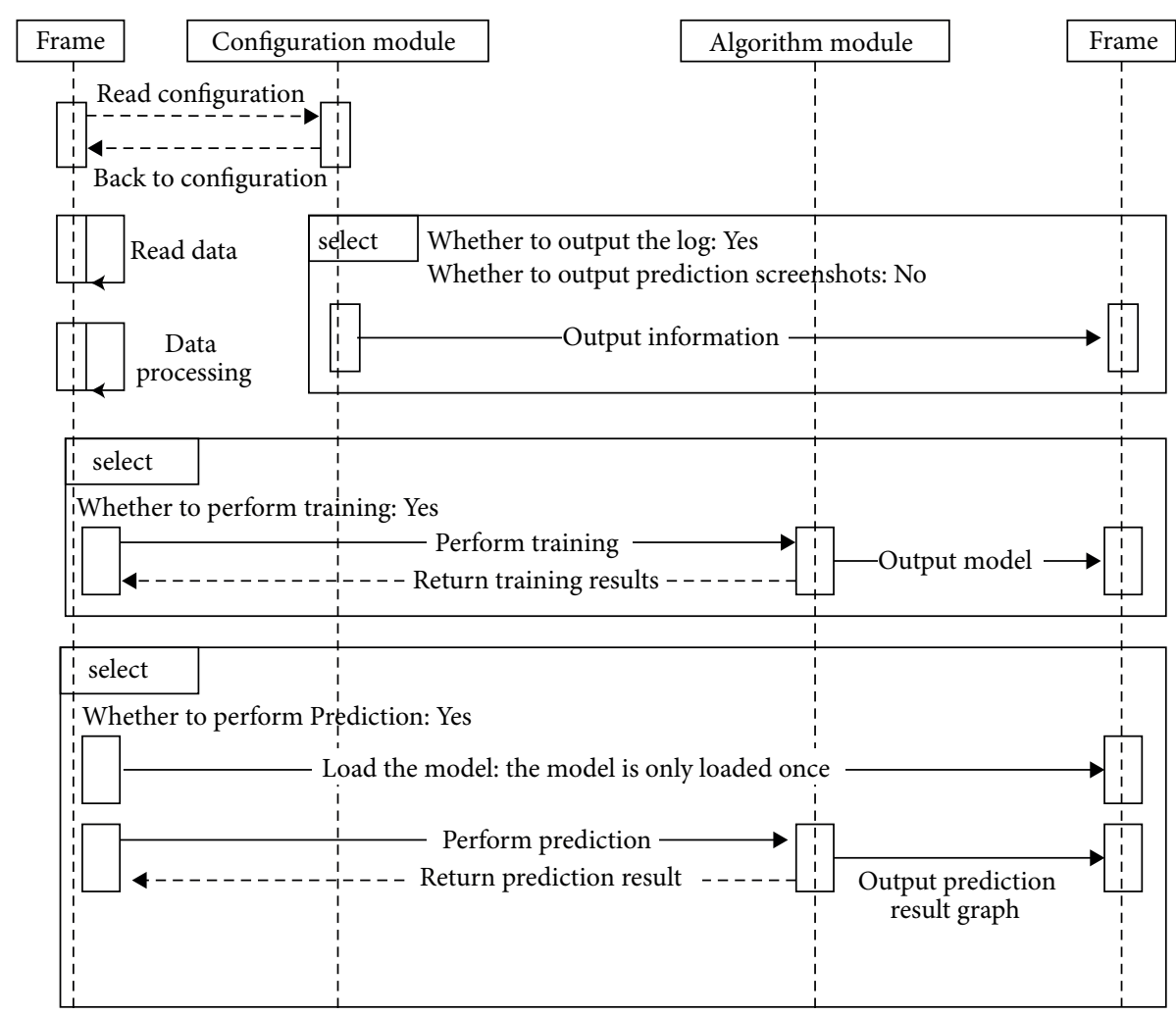

FIgURE 2: Bi-GRU-ATT model development framework diagram.

module, data processing module, and algorithm module, and the operation and modification of each module will not affect each other. First, read the algorithm type and algorithm parameters from the configuration module to determine the algorithm required by the model. This module does not need to modify the main frame, classify the external data, and store the training data in the specified directory according to the preprocessing method; according to the data source and different training methods and algorithms, save the training model to the automatically generated path, load the training model to execute prediction instructions, output the prediction result curve, and export the corresponding log file.

\section{Model Establishment}

3.1. Feature Selection. The construction conditions in the process of TBM tunneling are usually complex, often accompanied by a variety of uncertain factors that affect the prediction accuracy of TBM tunneling parameters. Therefore, it is necessary to analyze and extract the TBM tunneling parameter prediction model based on Bi-GRU. The TBM operating data contains 199-dimensional tunneling parameters, but some of them are greatly affected by human factors and have a poor linear correlation. Therefore, this paper only selects four indexes of the TBM complete excavation cycle section, namely, total thrust, penetration, cutterhead torque, and cutterhead power, as the output characteristics of the model.
The selection of input features is also of great significance to the prediction performance of the model, which can better reflect the operating status of the model under different working conditions and improve the prediction accuracy. Therefore, this paper uses Pearson correlation analysis to identify the 199-dimensional tunneling parameters of the complete tunneling cycle input by the model, eliminates irrelevant parameter variables, and selects key model parameters. The Pearson correlation coefficient is calculated as follows:

$$
\eta=\frac{\sum_{i=1}^{n}\left(X_{i}-\bar{X}\right)\left(Y_{i}-\bar{Y}\right)}{\sqrt{\sum_{i=1}^{n}\left(X_{i}-\bar{X}\right)^{2}} \sqrt{\sum_{i=1}^{n}\left(Y_{i}-\bar{Y}\right)^{2}}}
$$

where $\bar{X}$ is the average value of the data sample $X ; \bar{Y}$ is the average value of the data sample $Y$.

The Bi-GRU-based TBM tunneling parameter prediction model constructed in this paper uses the complete tunneling cycle data to predict the four parameters of the TBM complete excavation cycle section, namely, total thrust, penetration, cutterhead torque, and cutterhead power. According to the correlation analysis results, the 199-dimensional tunneling parameters of the complete tunneling cycle are sorted, and the 21 key parameters with the highest correlation with a certain TBM tunneling parameter to be predicted are extracted as the input features of the model, to improve the prediction accuracy of the model. The identification result of the input features of the model is shown in Figure 3. 

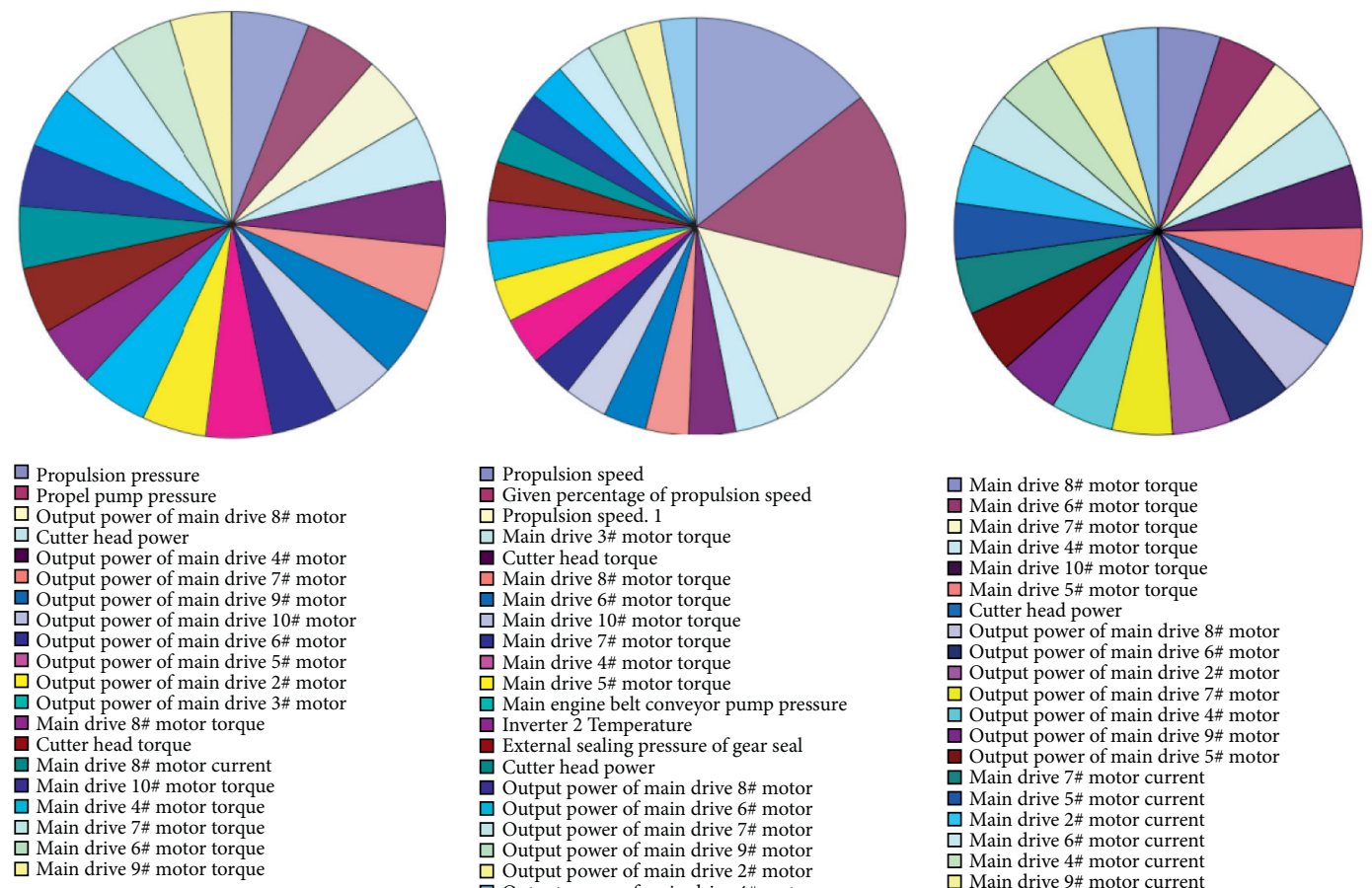

$\square$ Propulsion speed

Given percentage of propulsion speed

$\square$ Propulsion speed. 1

$\square$ Main drive 3\# motor torque

- Cutter head torque

$\square$ Main drive 8\# motor torque

Main drive $6 \#$ motor torque

$\square$ Main drive 10 \# motor torque

- Main drive $7 \#$ motor torque

$\square$ Main drive 4 m motor torque

$\square$ Main drive $5 \#$ motor torque

$\square$ Main engine belt conveyor pump pressure

Inverter 2 Temperature

External sealing pressure of gear sea

$\square$ Cutter head power

Output power of main drive 8\# motor

$\square$ Output power of main drive $6 \#$ motor

$\square$ Output power of main drive 7 \# motor

$\square$ Output power of main drive 9\# motor

$\square$ Output power of main drive $2 \#$ motor
$\square$ Output power of main drive $4 \#$ motor

(a)

(b)

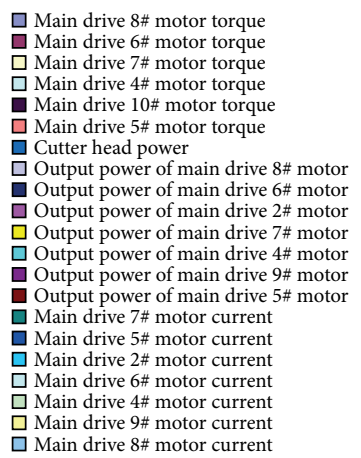

(c)

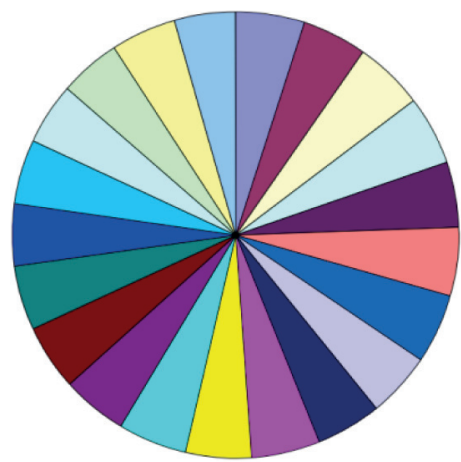

Output power of main drive 8\# motor

Output power of main drive 6\# moto

Output power of main drive $2 \#$ moto

Output power of main drive 7 \# moto

Output power of main drive $9 *$ motor

Output power of main drive 9 \# moto

Main drive 5\# motor torque

Main drive $4 \#$ motor torque

Main drive 10 motor torque

Main drive 6 m motor torque

Cutter head torque

Main drive 8\# motor torque

Main drive $5 \#$ motor current

Main drive $7 \#$ motor current

Main drive 2\# motor current

Main drive 6\# motor current

Main drive $4 \#$ motor current

Propulsion pressure

Total propulsion

(d)

Figure 3: Identification results of input features of Bi-GRU model. (a) Model identification result of total thrust. (b) Model identification result of penetration. (c) Model identification result of cutterhead torque. (d) Model identification result of cutterhead power.

3.2. Multipart Figures. In this paper, the attention mechanism is used as a layer of the Bi-GRU model to learn the output of the model in a serial manner, giving full play to the important local feature extraction capabilities of the attention model and the sequential feature processing capabilities of the Bi-GRU model. The intelligent prediction model of TBM tunneling parameters based on Bi-GRU constructed in this paper is shown in Figure 4, which is mainly composed of five parts: input layer, Bi-GRU layer, attention mechanism, fully connected layer, and output layer. 


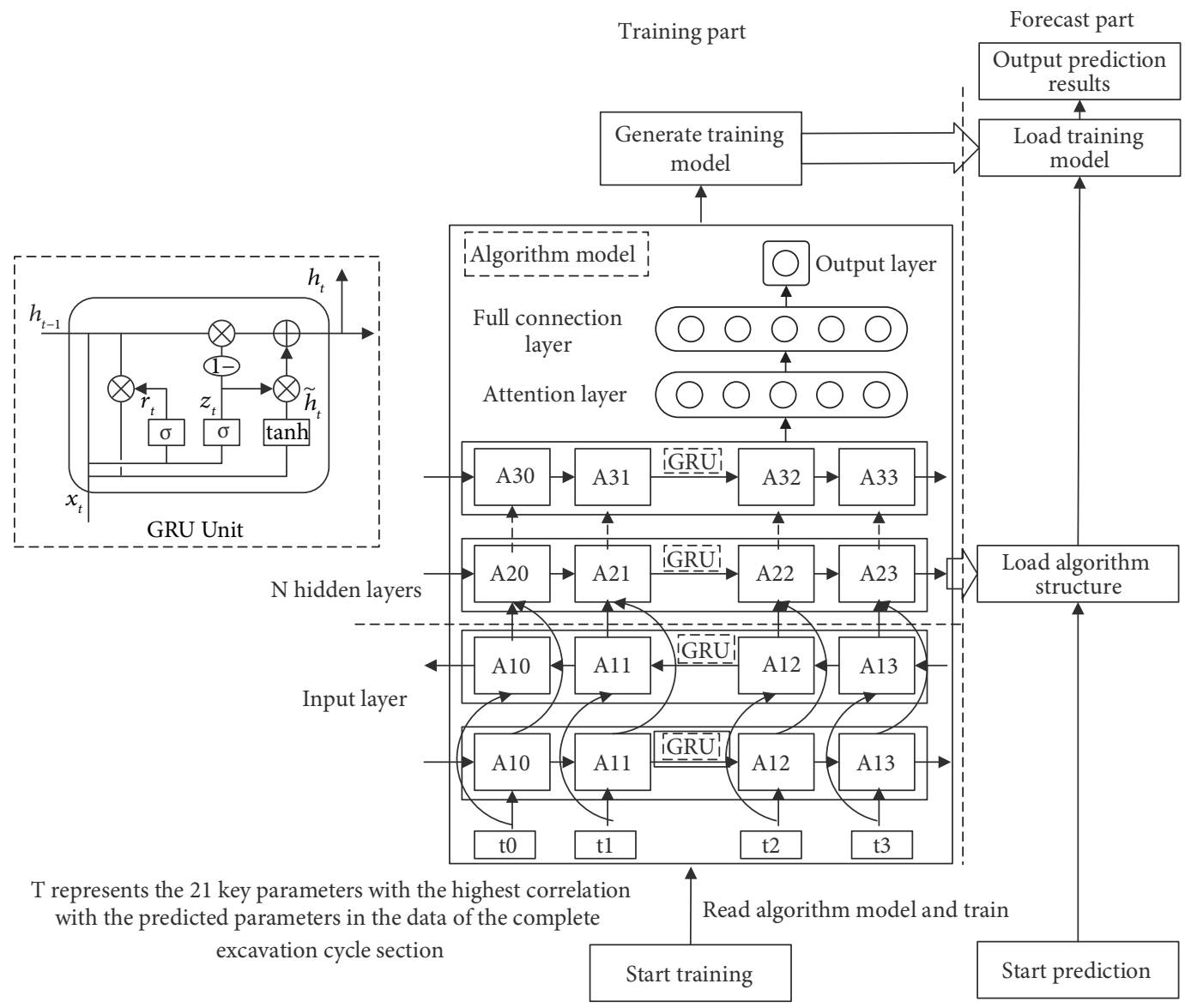

FIGURE 4: TBM tunneling parameter prediction model based on Bi-GRU-ATT.

(1) Input layer. After the original data is preprocessed by Pearson correlation analysis method, triple standard deviation method, and other data processing techniques, 21 key parameters with the highest correlation with the prediction parameters are selected as the input vector of the model and input to the BiGRU layer according to the Pearson correlation analysis results.

(2) Bi-GRU layer. Input the input vector to the forward propagation GRU layer and the backpropagation GRU layer, respectively. The two parallel layers mainly characterize the correlation between sequence data and generalize the input of each layer. By updating the gate to control the extent to which the state information at the previous moment is brought into the current state, the input features are trained along the forward sequence and the reverse sequence, respectively, and the training vectors of the forward and reverse sequences are synthesized to obtain the output vector of the Bi-GRU layer.

(3) Attention mechanism and fully connected layer. The attention layer performs weighting operations on the output vector processed by Bi-GRU, then outputs it to the fully connected layer, and maps the distributed features to the sample label space.

(4) Output layer. Output predicted values, namely, total thrust, penetration, cutterhead torque, and cutterhead power in the complete driving cycle section of TBM.

The specific algorithm flow of the Bi-GRU-ATT prediction model is as follows: first, the data set is divided into a training set and a test set at a ratio of $17: 1$. The training set is used to train the model and optimize the model parameters; the test set is used to predict the TBM tunneling parameters; the 21 key parameters with the highest correlation in the data of the complete tunneling cycle section are selected as the input of the input layer, and the basic structure and hyperparameters of the Bi-GRU-ATT model are set, including time step and iteration times. Adam optimizer is used to train the network model, and the mean square error (MSE) function is used as the loss function to automatically adjust model weights, iteratively optimize model parameters, generate and load model files, and output model prediction results. Finally, an appropriate evaluation function is selected to evaluate the prediction effect of the model and to verify the prediction performance of the model in this paper. 


\section{Engineering Data Set}

4.1. Project Overview. The TBM3 bid section of the Jilin Water Supply Project is the control bid section with the longest single construction section, the most unexpected geological disasters, and the most difficult construction in the entire central water diversion project in Jilin Province. It is located between Chalu River and Wende River in Yongji County, Jilin City, with a total length of $22.955 \mathrm{~km}$. Tunnel excavation is mainly based on full-face TBM construction, of which the TBM construction section is $20.198 \mathrm{~km}$ long. The stratigraphic lithology involved in this section can be roughly divided into limestone and granite, which are mainly distributed between grades II and V. According to the statistics of geological data, the proportion of the surrounding rock grade (grades II to V) of the construction section is $5.9 \%, 66.13 \%, 23.72 \%$, and $4.25 \%$, respectively; the distribution ratio of limestone area to granite area is $1.58: 1$. As shown in Figure 5(a), the proportions of four types of the surrounding rock grade in limestone are 5.19\%, 66.70\%, $22.83 \%$, and $5.28 \%$, respectively. Figure 5(b) shows that the proportions of the surrounding rock grade in granite are $7.03 \%, 65.23 \%, 25.14 \%$, and $2.6 \%$.

The data in this paper are selected from a construction section in the TBM3 bid section, with a total length of $7.5 \mathrm{~km}$. TBM operating data are collected in second, and 86400 pieces of data are collected in a day, including 199dimensional tunneling parameters such as total thrust, penetration, cutterhead torque, and cutterhead power. In this project, TBM construction usually divides the tunneling cycle according to the cylinder stroke, with about $10 \sim 20$ tunneling cycles per day. Among them, the variation curve of TBM tunneling parameters on a certain day is shown in Figure 6.

4.2. Tunneling Cycle Extraction. It can be seen from Figure 6 that TBM is not always in the working state, and there is a huge amount of shutdown section data that cannot be used for machine learning between each tunneling cycle. Therefore, it is necessary to determine the working state of TBM at each time by constructing a binary state function to extract the complete tunneling cycle data. The judgment formula [38] is as follows:

$$
\begin{aligned}
I & =f(N) f(F) f(T) f(V), \\
f(x) & =\left\{\begin{array}{l}
1, x \neq 0 \\
0, x=0
\end{array}\right.
\end{aligned}
$$

where $N$ is the speed of the cutter head; $F$ is the total thrust; $T$ is the cutterhead torque; $V$ is the propulsion speed; $I$ is the state discrimination function.

When $I=1$, it is determined that the TBM is in the tunneling state, and when $=0$, it is judged that TBM is in the shutdown state. According to the judgment results of the TBM working status at each time, valid data samples are extracted. Each group of continuous tunneling sequences corresponds to a complete tunneling cycle, and the data between adjacent tunneling cycles corresponds to a set of shutdown data. As shown in Figure 7, the TBM tunneling process can be divided into an ascending section and a complete tunneling cycle.

In the traditional TBM tunneling process, the operator generally predicts the parameters of the complete tunneling cycle according to the data change law of the ascending section [39]. Due to the complexity of the TBM tunneling construction environment, the driver needs to spend a lot of time identifying and extracting the ascending section data to adjust the TBM operating state, and the effective tunneling efficiency is low. To ensure safe and efficient tunneling of TBM, reduce redundant operations during the driver's operation, and optimize and adjust the current tunneling parameters, this paper selects the data of the complete tunneling cycle section to predict the four tunneling parameters, namely, the total thrust, penetration, cutterhead torque, and cutterhead power in the complete driving cycle section of TBM. Figure 8 shows the comparison between the traditional ascending segment prediction method and the full-period prediction method.

4.3. Data Processing. Due to a large amount of invalid data in the field engineering data, it is necessary to preprocess the original data in the database to extract valid data samples. First, it is necessary to identify and read the original data in the database, including the total thrust, cutterhead torque, and other tunneling parameters, and judge the data. Take the total thrust as an example; the first non-zero data of the total thrust in the input original data needs to be found and marked as P1, and then the second data with zero of the total thrust is found and marked as P2. Subsequently, it needs to be analyzed and judged whether the number of rows between data P1 and data $\mathrm{P} 2$ is within the range of [ $500 \mathrm{~s}, 5000 \mathrm{~s}]$. If so, the data will be discarded; otherwise, the data will be output to the specified file, and each file can have a maximum of 300 cycles. According to this method, all the data are read successively until all the effective data are extracted, and the data processing process is completed. The specific data processing flow is shown in Figure 9.

In the process of data processing, affected by the construction environment, construction equipment, and construction experience of construction personnel during the TBM tunneling process, there are many abnormal values in the original data, which affect the prediction effect of the model. To eliminate the above-mentioned abnormal working data, the original data was processed by the triple standard deviation method. Taking the four parameters to be predicted in this paper as an example, it is necessary to calculate the mean $\mu$ and standard deviation $\sigma$ of the four tunneling parameters in the entire tunneling progress. Then, it is judged whether the distance between the parameter value and the mean value is greater than $\mu+3 \sigma$; if it is greater, it is regarded as an abnormal value and replaced by the average value of 5 data points near the abnormal value.

$$
X-\mu \geq=3 \sigma
$$

where $X$ represents the predicted data sample; $\mu$ represents the sample mean; $\sigma$ represents the sample standard deviation. 


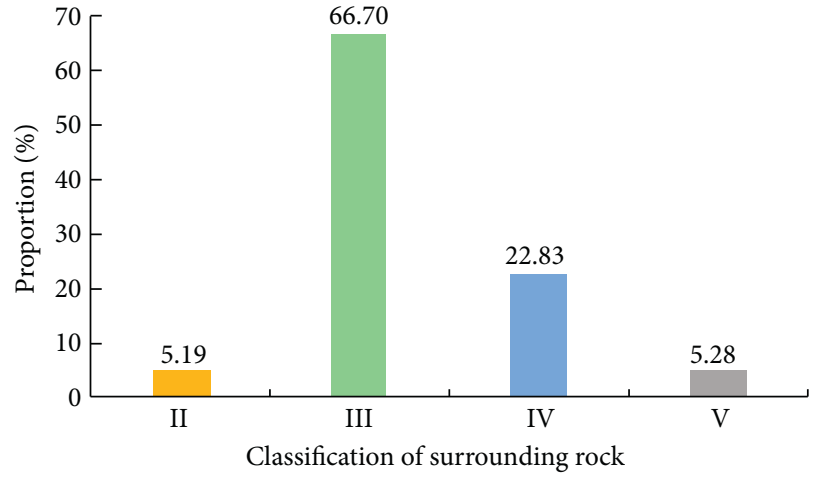

(a)

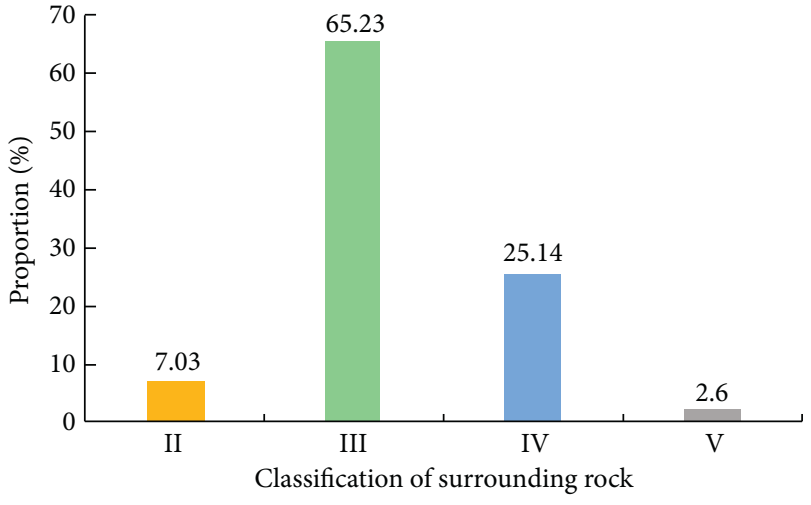

(b)

FIGURE 5: Engineering geological conditions. (a) Limestone classification statistics. (b) Granite classification statistics.

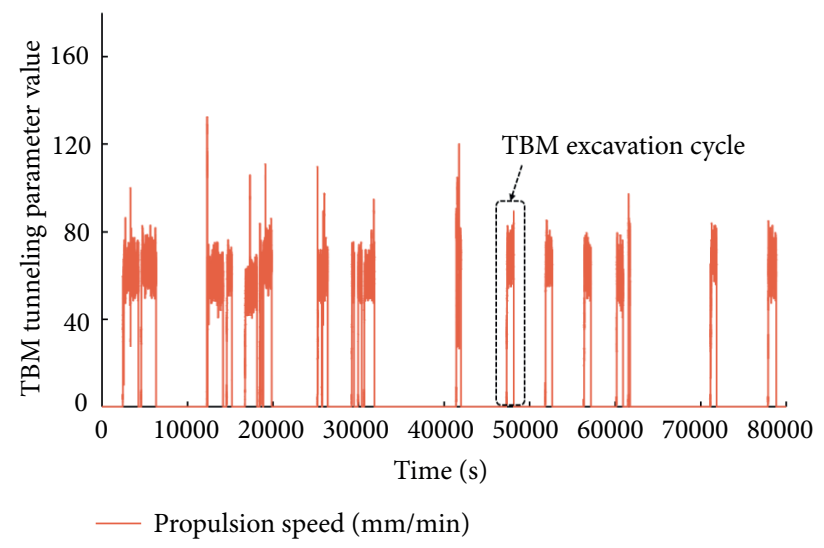

FIGURE 6: Variation curve of TBM tunneling parameters on a certain day.

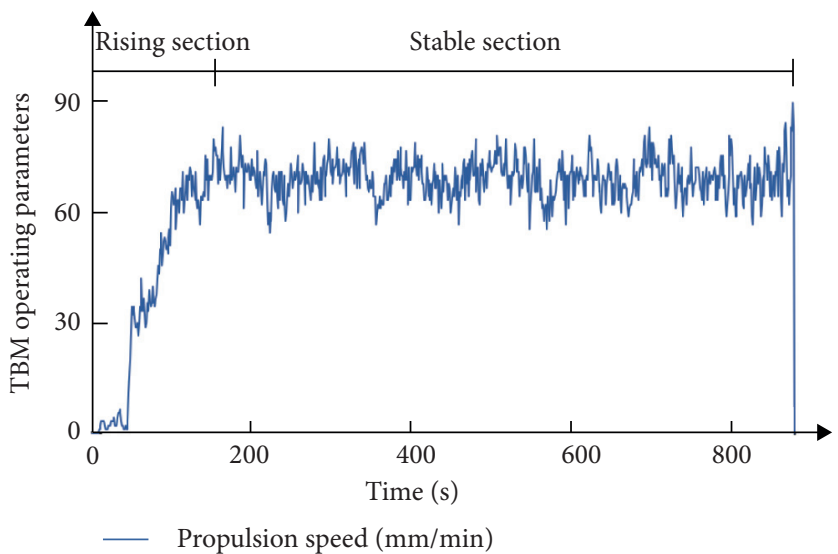

FIgURE 7: A complete tunneling cycle in TBM construction. 


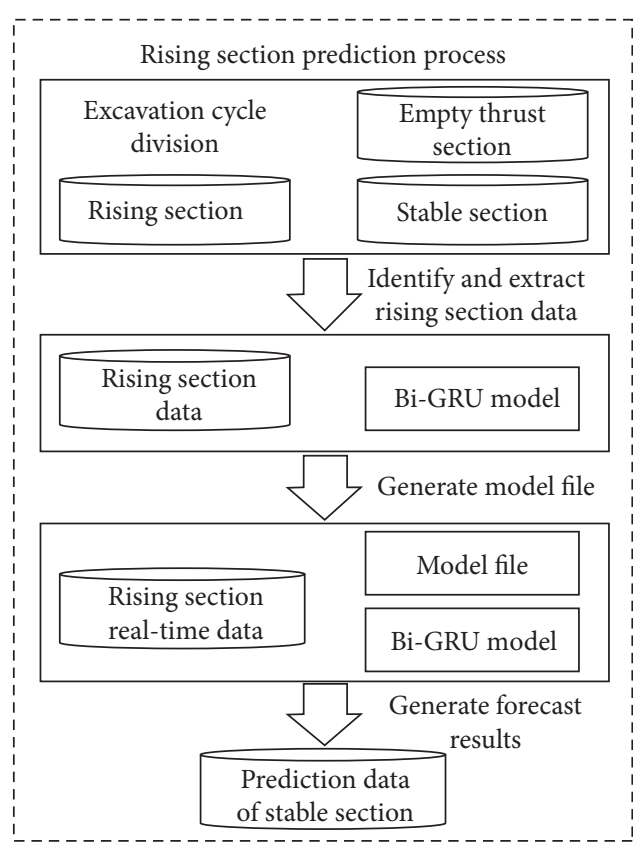

(a)

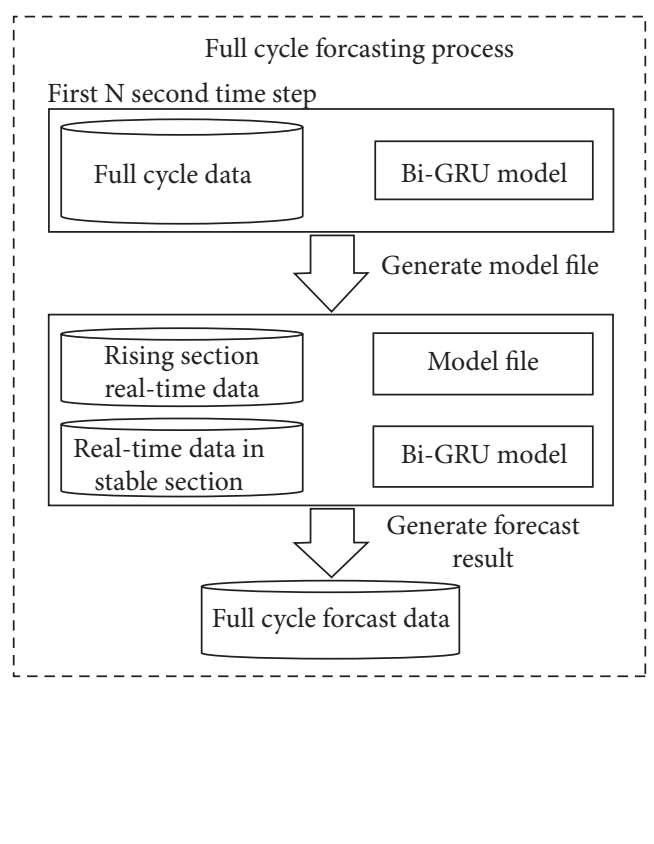

(b)

FIGURE 8: Comparison of complete tunneling cycle and ascending section. (a) Ascending segment prediction. (b) Full-period prediction.

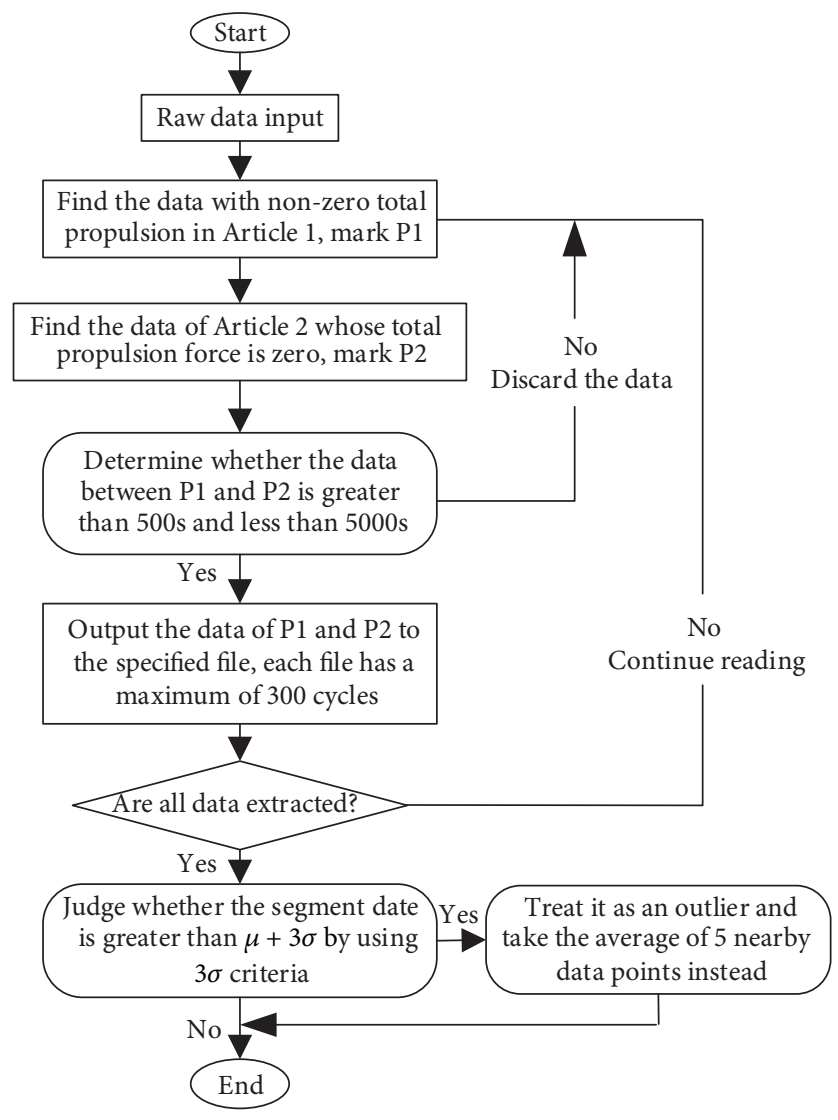

Figure 9: Data processing flowchart. 
In addition, to eliminate the influence of dimensional differences in the data, the data samples need to be normalized before model training [40]:

$$
x^{*}=\frac{x-x_{\min }}{x_{\max }-x_{\min }}
$$

where $x^{*}$ represents the normalized data; $x_{\max }$ and $x_{\min }$ represent the maximum and minimum value of the data, respectively.

\section{Model Application and Comparison}

5.1. Model Evaluation Index. In order to verify the prediction effect of the model, four evaluation indexes commonly used in regression analysis are used to quantify the estimation error of the model, namely, mean absolute percentage error (MAPE), goodness of fit $\left(R^{2}\right)$, mean absolute error (MAE), and root-mean-square error (RMSE), and the calculation formula is as follows:

$$
\begin{aligned}
\text { MAPE } & =\frac{1}{n} \sum_{i=1}^{n} \frac{\left|y_{i}^{*}-y_{i}\right|}{y_{i}}, \\
R^{2} & =1-\sqrt{\frac{\sum_{i-1}^{n}\left(y_{i}-y_{i}^{*}\right)^{2}}{\sum_{i=1}^{n} y_{i}^{2}}}, \\
\operatorname{MAE} & =\frac{1}{n} \sum_{i=1}^{n}\left|y_{i}^{*}-y_{i}\right| \operatorname{RMSE}=\sqrt{\frac{1}{n} \sum_{i=1}^{n}\left(y_{i}^{*}-y_{i}\right)^{2},}
\end{aligned}
$$

where $y_{i}$ is the true value; $y_{i}^{*}$ is the predicted value of the model; $i$ is the sample number; $n$ is the total number of samples in the test set.

5.2. Model Application. According to the results of data processing, the nonworking status and abnormal data are eliminated, and the parameters of the complete tunneling section are averaged to obtain the data set for modeling. To improve the training model to learn the data operation law, improve the generalization ability of the model, and prevent the model from overfitting, it is necessary to increase the model training sample. Therefore, the sample set and the test sample set are divided into training according to $17: 1$. The total number of samples is 9900, and the number of training samples and the number of test samples are 9350 and 550, respectively. The training samples are used to train the model and optimize the model parameters; the test samples are used for prediction, and an appropriate evaluation function is selected to verify the prediction effect of the model. The 21 key parameters in the complete tunneling cycle that has the highest correlation with a certain tunneling parameter to be predicted are selected as the input parameters of the Bi-GRU-ATT model. The four key parameters, namely, the total thrust, penetration, cutterhead torque, and cutterhead power, are tested and verified. Figure 10 shows the prediction results of the stable section in the complete excavation cycle section of the selected $\mathrm{Bi}$ GRU-ATT model with four excavation parameters.

It can be seen from the comparison curve between the predicted value and the measured value in Figure 10 that the predicted values of the four TBM tunneling parameters are in good agreement with the measured values, and the change of the predicted value curve is less than that of the measured value curve, indicating that this model can better predict the tunneling parameters of TBM complete tunneling cycle. To evaluate the prediction accuracy of the network model, it is necessary to analyze and predict the goodness of fit $R^{2}$ and MAPE of the four tunneling parameters. By calculating the prediction samples, it can be seen from Table 1 that the MAPE of each parameter is within 1.8\%, and the goodness of fit $R^{2}$ is also above 0.92 , which shows that the prediction model has good network generalization promotion ability and prediction accuracy and has certain guiding significance for the judgment of TBM tunneling state and the optimization and adjustment of parameters.

5.3. Model Comparison. A set of data samples are used to test the performance of Bi-GRU, GRU, and LSTM, and their errors are compared and analyzed. Figures 11-13 are the prediction result curves of the stable section in the complete excavation cycle section of the selected three models. It can be seen from the prediction results that the overall trend of the predicted value change curve and the actual measured value change curve in Bi-GRU, GRU, and LSTM models is the same, but there will be a large deviation from the actual measured value in some samples. Among them, the prediction of total thrust and penetration is in good agreement with the actual measurement results; the prediction of cutterhead torque and cutterhead power is relatively low in agreement with the actual measurement results.

For the sake of quantitatively analyzing and comparing the prediction performance of the four models, MAPE and the goodness of fit $R^{2}$ are used as the evaluation indexes to measure the prediction accuracy of the model. The comparison of prediction results of different models is shown in Table 2. The prediction accuracy of the four models for TBM tunneling parameters is Bi-GRU-ATT, Bi-GRU, GRU, and LSTM from high to low.

It can be seen from the comparison in Table 2 that the bidirectional cyclic neural network is better than the unidirectional cyclic neural network with the same structure; that is, the Bi-GRU network is better than the GRU network. As a cyclic neural network, GRU neural network has memory capabilities and can effectively process input data with time series characteristics, thereby accurately predicting the tunneling parameters of the complete tunneling cycle. The Bi-GRU neural network is equivalent to adding a backpropagation layer on the basis of the GRU neural network, which controls the forward and backward output, respectively, making full use of the time sequence information before and after the sample data, and reducing the error.

It can be seen from Table 2 that the Bi-GRU-ATT model has achieved the best prediction effect, with a 


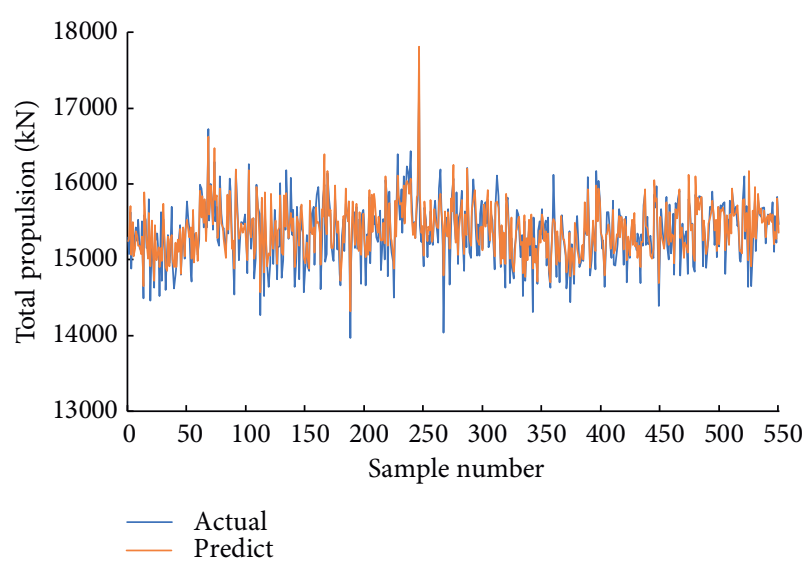

(a)

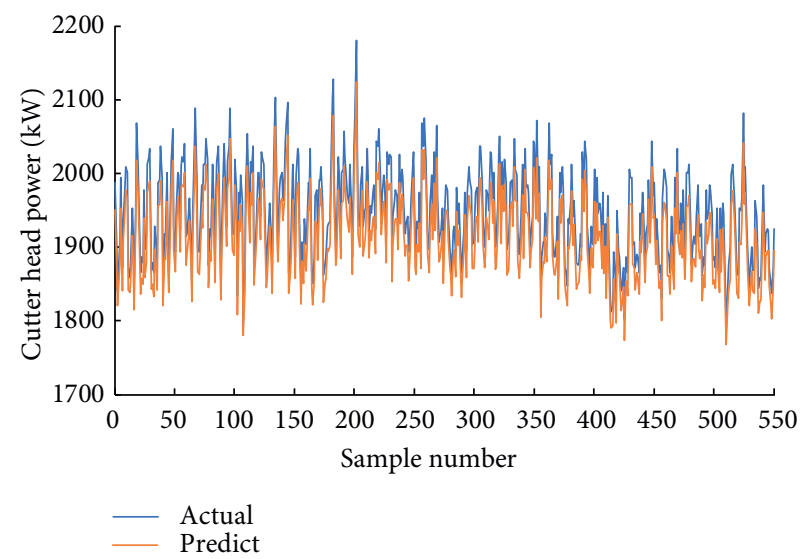

(c)

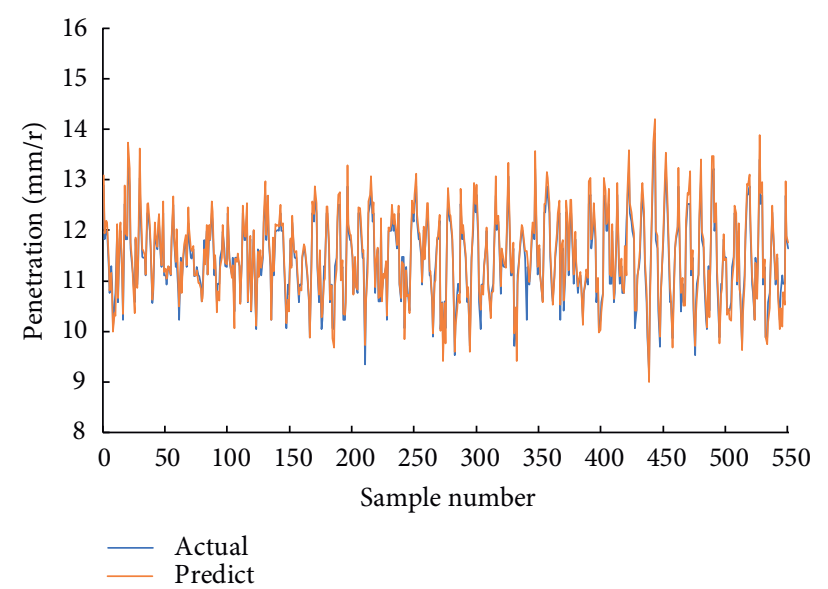

(b)

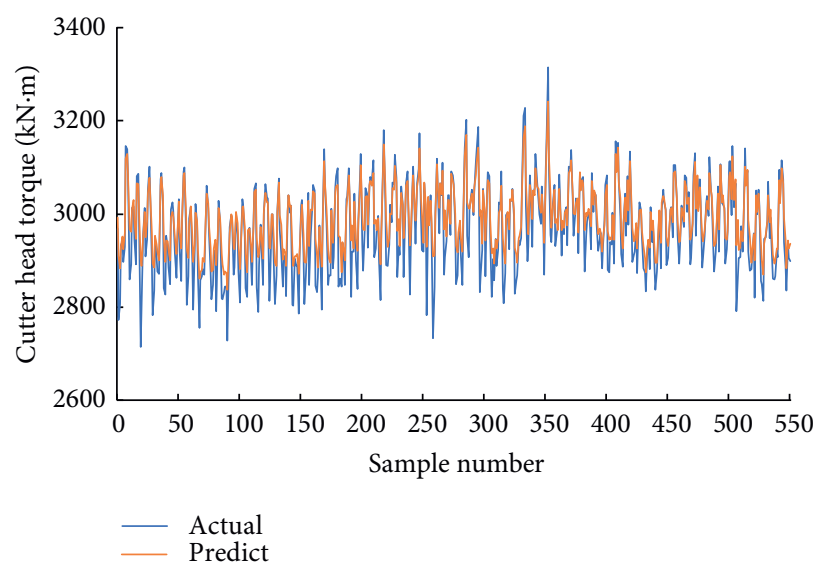

(d)

Figure 10: Prediction results of Bi-GRU-ATT model. (a) Prediction results of total thrust F. (b) Prediction results of penetration $e$. (c) Prediction results of cutterhead power P. (d) Prediction results of cutterhead torque $T$.

TABLE 1: Evaluation indexes of prediction results of Bi-GRU-ATT model.

\begin{tabular}{|c|c|c|c|c|c|}
\hline Model & Parameter & MAPE (\%) & $R^{2}$ & MAE & RMSE \\
\hline \multirow{4}{*}{ Bi-GRU-ATT } & Total thrust & 1.01 & 0.925 & 151.52 & 193.62 \\
\hline & Penetration & 1.60 & 0.930 & 0.15 & 0.20 \\
\hline & Cutterhead torque & 1.40 & 0.936 & 31.30 & 44.44 \\
\hline & Cutterhead power & 1.71 & 0.944 & 34.13 & 34.75 \\
\hline
\end{tabular}

goodness of fit $R^{2}$ of up to 0.944 , and the minimum MAPE is $1.01 \%$. Compared with the Bi-GRU model without attention mechanism, the highest goodness of fit $R^{2}$ is improved by up to 0.06 . This shows that when the attention mechanism is added to the model, the prediction accuracy will be improved to a certain extent, which verifies the excellent performance of the attention mechanism in the TBM tunneling parameters. In addition, the prediction accuracy of unidirectional GRU is similar to that of unidirectional LSTM, but unidirectional GRU is better than unidirectional LSTM. The reason is that the unidirectional GRU only has two gate control structures, the update gate and the reset gate, which not only make its output faster than LSTM and have better performance, but also can effectively avoid the overfitting phenomenon of the model in non-high-dimensional prediction tasks.

To sum up, the Bi-GRU-ATT intelligent prediction model established in this paper has stronger learning and prediction capabilities than the other three recurrent neural network prediction models and provides a more feasible auxiliary intelligent decision-making method for TBM safe and efficient tunneling construction.

5.4. Engineering Application Effect Analysis. In the traditional TBM tunneling construction, the selection of tunneling parameters basically relies on human experience to make judgments and adjustments. The matching of tunneling parameters and rock mass state parameters is poor. Once complicated geological conditions are encountered, it is 


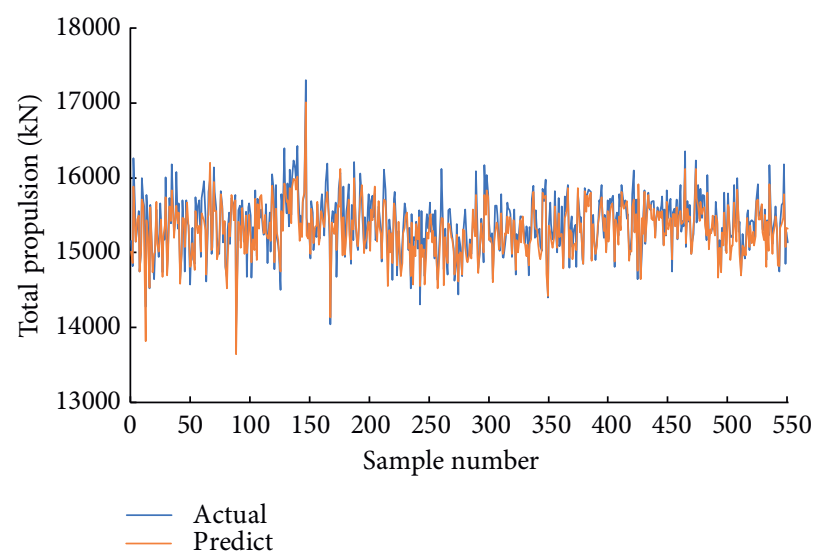

(a)

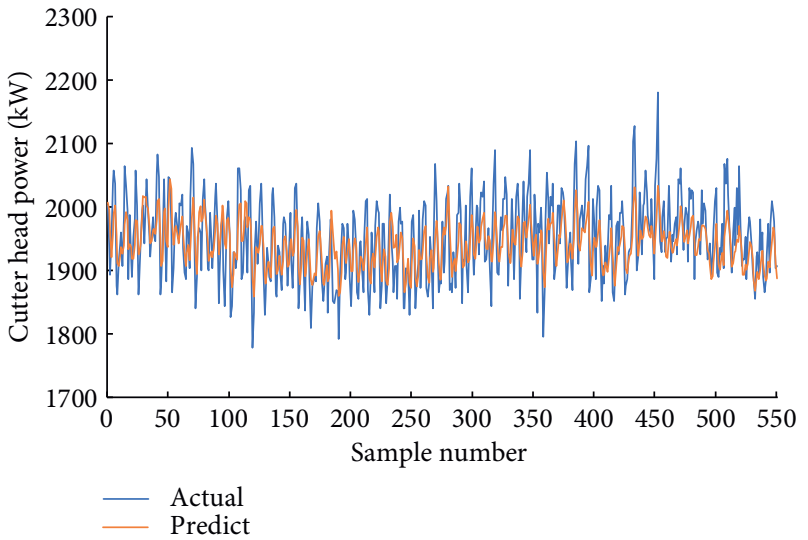

(c)

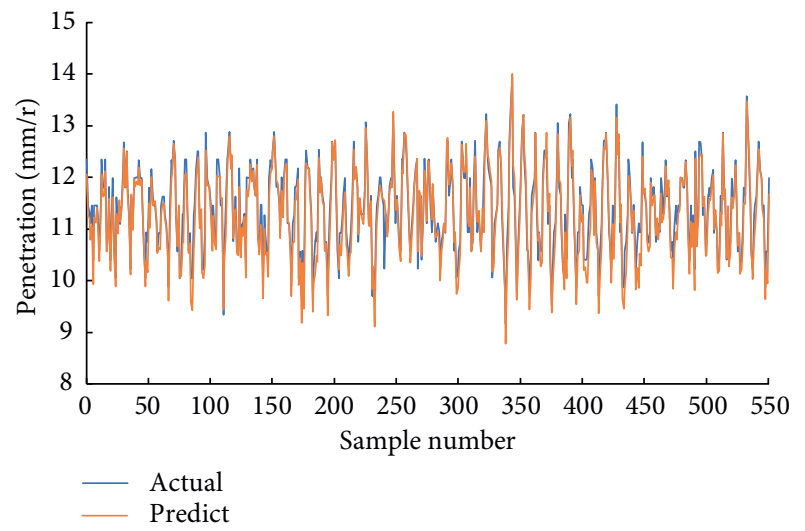

(b)

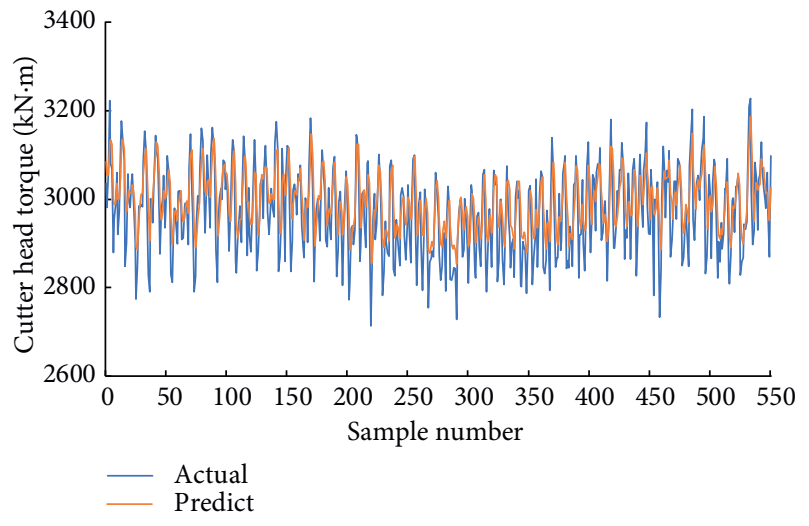

(d)

Figure 11: Prediction results of Bi-GRU model. (a) Prediction results of total thrust $F$. (b) Prediction results of penetration $e$. (c) Prediction results of cutterhead power $P$. (d) Prediction results of cutterhead torque $T$.

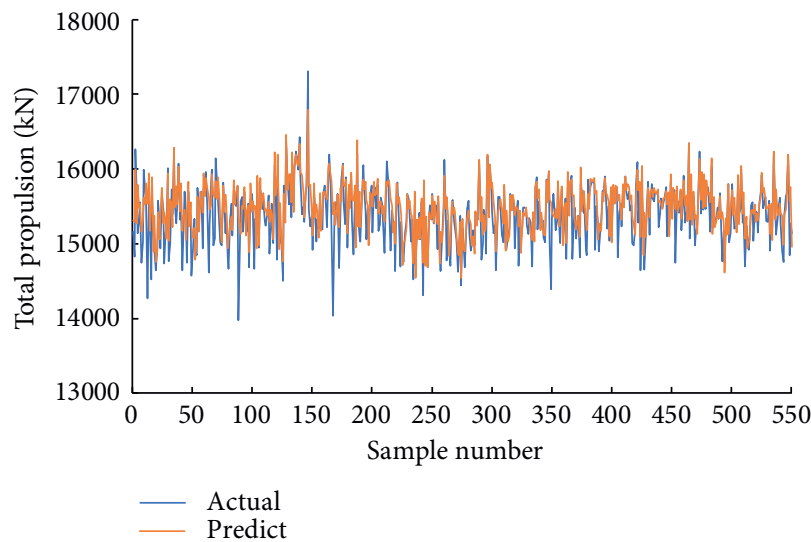

(a)

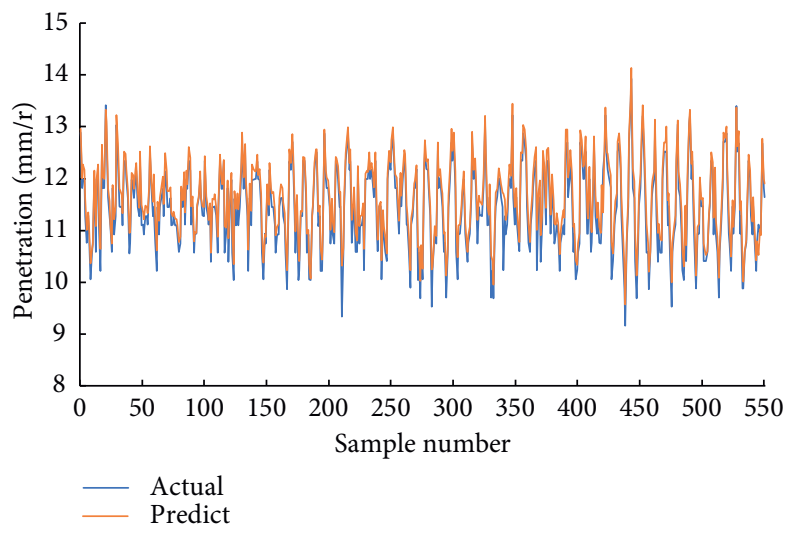

(b)

Figure 12: Continued. 


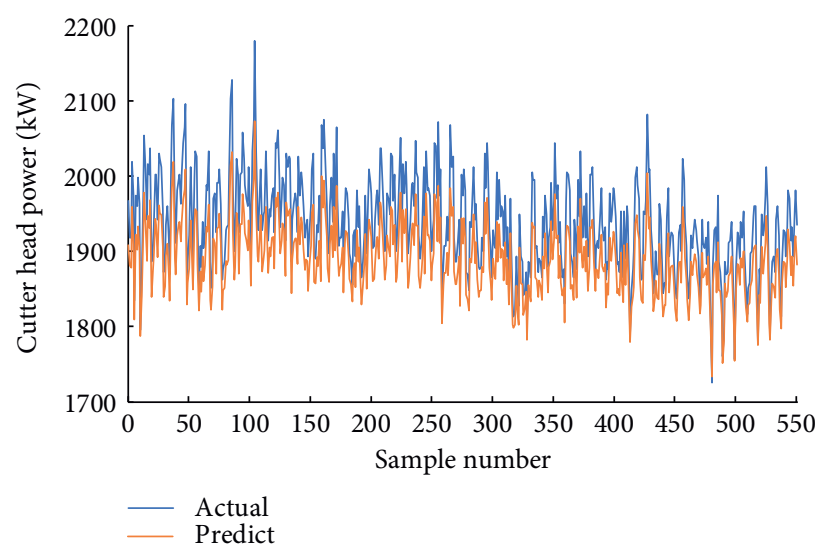

(c)

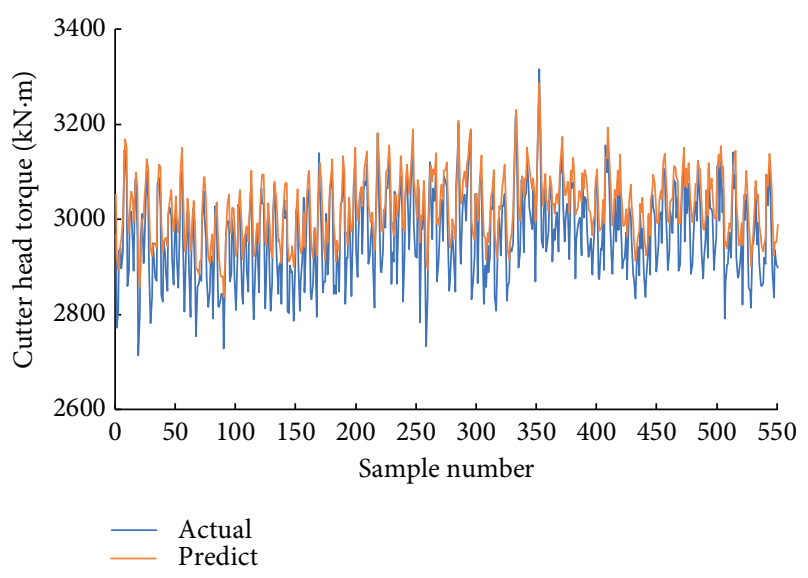

(d)

Figure 12: Prediction results of GRU model. (a) Prediction results of total thrust $F$. (b) Prediction results of penetration $e$. (c) Prediction results of cutterhead power $P$. (d) Prediction results of cutterhead torque $T$.

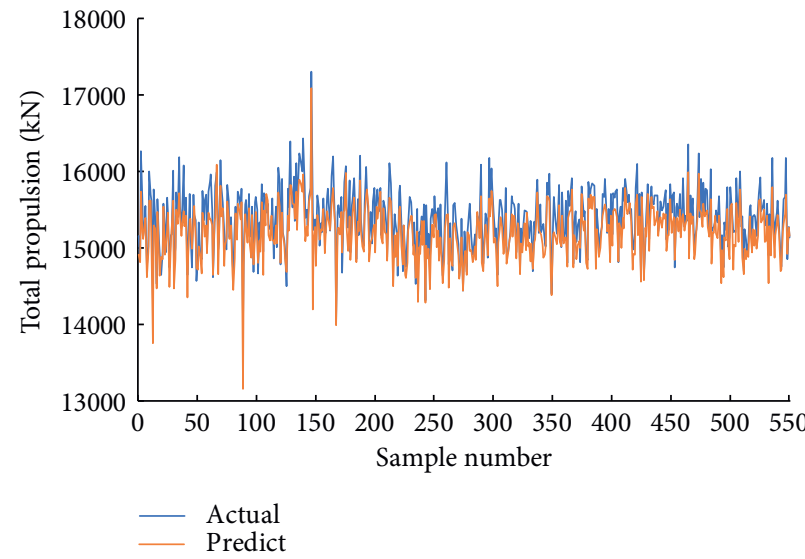

(a)

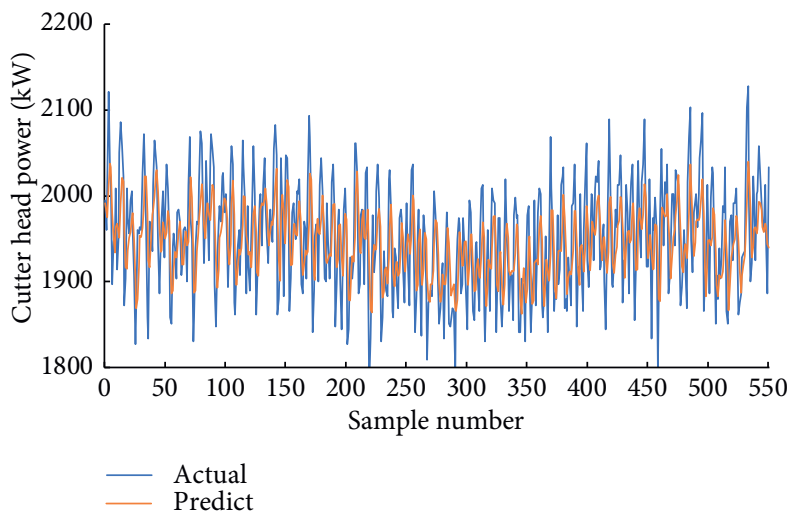

(c)

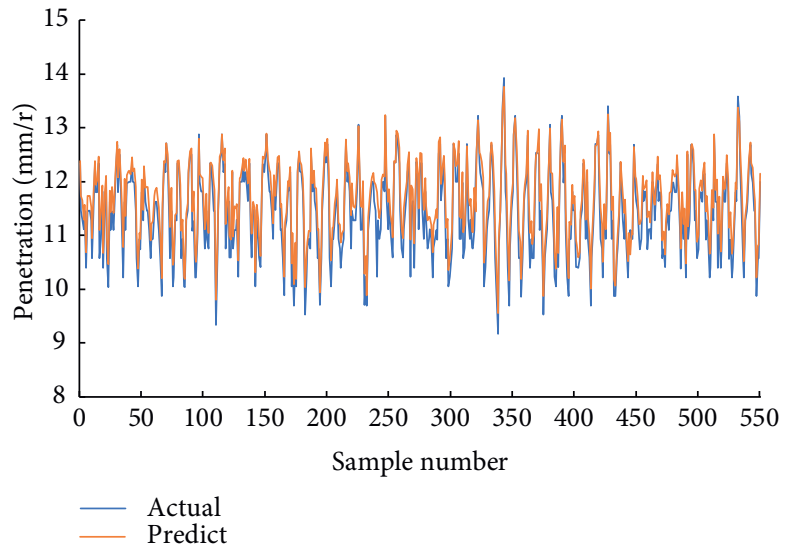

(b)

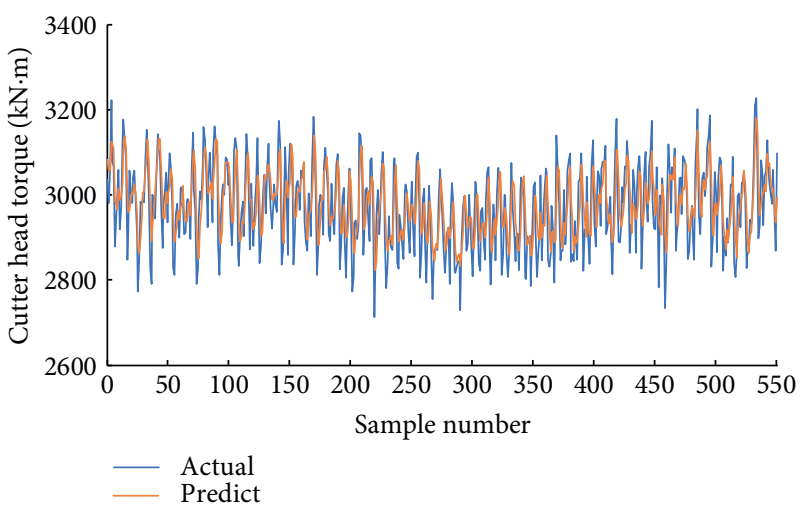

(d)

Figure 13: Prediction results of LSTM model. (a) Prediction results of total thrust $F$. (b) Prediction results of penetration $e$. (c) Prediction results of cutterhead power $P$. (d) Prediction results of cutterhead torque $T$.

difficult to adjust the tunneling state in a timely and effective manner. With the rapid development of computer technology, artificial intelligence technology is gradually applied to TBM tunnel construction. Compared with traditional methods, one of the main advantages of artificial intelligence technology is a high degree of self-programming, which means that manual supervision of this process is no longer necessary, saving a lot of labor costs. The Bi-GRU-ATT model 
TABle 2: Comparison of evaluation indexes of prediction results among different recurrent neural network models.

\begin{tabular}{|c|c|c|c|c|c|}
\hline Model & Parameter & MAPE (\%) & $R^{2}$ & MAE & RMSE \\
\hline \multirow{4}{*}{$\begin{array}{l}\text { Bi-GRU- } \\
\text { ATT }\end{array}$} & Total thrust & 1.01 & 0.925 & 151.52 & 193.62 \\
\hline & Penetration & 1.60 & 0.930 & 0.15 & 0.20 \\
\hline & $\begin{array}{l}\text { Cutterhead } \\
\text { torque }\end{array}$ & 1.40 & 0.936 & 31.30 & 44.44 \\
\hline & $\begin{array}{c}\text { Cutterhead } \\
\text { power }\end{array}$ & 1.71 & 0.944 & 34.13 & 34.75 \\
\hline \multirow{4}{*}{ Bi-GRU } & Total thrust & 1.11 & 0.915 & 146.35 & 184.23 \\
\hline & Penetration & 2.05 & 0.898 & 0.20 & 0.26 \\
\hline & $\begin{array}{c}\text { Cutterhead } \\
\text { torque }\end{array}$ & 1.84 & 0.909 & 52.65 & 67.00 \\
\hline & $\begin{array}{c}\text { Cutterhead } \\
\text { power }\end{array}$ & 2.13 & 0.884 & 38.30 & 46.02 \\
\hline \multirow{4}{*}{ GRU } & Total thrust & 1.21 & 0.859 & 196.68 & 280.99 \\
\hline & Penetration & 2.47 & 0.867 & 0.23 & 0.27 \\
\hline & $\begin{array}{c}\text { Cutterhead } \\
\text { torque }\end{array}$ & 2.26 & 0.879 & 60.87 & 73.37 \\
\hline & $\begin{array}{c}\text { Cutterhead } \\
\text { power }\end{array}$ & 2.52 & 0.870 & 48.41 & 50.95 \\
\hline \multirow{4}{*}{ LSTM } & Total thrust & 1.44 & 0.870 & 205.60 & 252.02 \\
\hline & Penetration & 2.97 & 0.840 & 0.27 & 0.32 \\
\hline & $\begin{array}{l}\text { Cutterhead } \\
\text { torque }\end{array}$ & 2.04 & 0.889 & 55.88 & 67.97 \\
\hline & $\begin{array}{c}\text { Cutterhead } \\
\text { power }\end{array}$ & 2.7 & 0.860 & 44.61 & 52.89 \\
\hline
\end{tabular}

proposed in this study uses an adaptive algorithm to automatically adjust the processing method, processing sequence, and boundary conditions in the data processing and analysis stage according to the characteristics of the preprocessed data, so as to make it consistent with the structural characteristics and statistics of the processed data. The distribution characteristics are adapted to ensure the extraction of the rockmachine interaction relationship under real-time rock mass conditions, so as to realize the real-time prediction of the tunneling performance of the model under the given TBM control parameters, in order to promote the intelligent development of TBM tunneling; in addition, the Bi -GRU-ATT model can reflect the current rock mass state through the change law of the excavation parameter value in the complete excavation cycle and adjust and optimize the current excavation parameters in real time according to the current rock mass state information to ensure safe and efficient excavation of TBM. Although this model is based on a huge amount of construction data of TBM tunnel projects that have been built or under construction, the unknown rock mass status information still needs to be obtained through on-site drilling core sampling, in situ experiments, etc.; the artificial intelligence technology represented by this model is only used as an example. This kind of auxiliary intelligent decision-making method, the steps of planning of the excavation task, and control of the excavation state in the excavation process still inevitably require human intervention. Therefore, to further improve the intelligent prediction model of TBM tunneling parameters to adapt to the TBM tunneling construction of different engineering geological conditions is a problem that we urgently need to solve.

\section{Conclusions}

This paper proposes a prediction method of TBM tunneling parameter based on the Bi-GRU-ATT model and selects the normalized complete tunneling cycle section data to predict the tunneling parameters of the complete tunneling cycle. The training set and test set are divided by the operation data of the TBM3 bid section of the Jilin Water Supply Project, the nonworking state and abnormal data are eliminated, and the complete excavation cycle is extracted. Based on Pearson correlation analysis, the 199-dimensional tunneling parameters selected from the complete tunneling cycle are sorted, and the 21 key parameters with the highest correlation with a certain TBM tunneling parameter to be predicted are extracted as the input features of the model, so as to predict four TBM tunneling parameters (total thrust, penetration, cutterhead torque, and cutterhead power) in the complete driving cycle section. The advantages of this model are verified by comparing the Bi-GRUATT model with Bi-GRU, unidirectional LSTM, and unidirectional GRU models. The main conclusions are as follows:

(1) Bi-GRU-ATT is trained based on the complete cycle data of the TBM tunneling cycle, and the intelligent prediction method based on the Bi-GRU-ATT model is constructed, which can effectively predict four key TBM tunneling parameters. The average absolute percentage error (MAPE) of the prediction results of each parameter is less than $1.8 \%$, and the goodness of fit $R^{2}$ is greater than 0.92 , which verifies the accuracy and effectiveness of this model. The results show that this model has a good guiding significance for realizing the real-time prediction of TBM tunneling parameters and assisting the optimization and adjustment of TBM tunneling parameters.

(2) Compared with the existing methods of using ascending section data of TBM tunneling cycle to predict the tunneling parameters of the stable section, this paper selects the data of complete tunneling cycle section to predict the tunneling parameters of the complete tunneling cycle, which can significantly improve the complexity and difficulty of data processing in the TBM tunneling process and then greatly improve the preprocessing efficiency of massive TBM tunneling data.

(3) Comparing and analyzing the prediction results of recurrent neural network models, it can be seen that the Bi-GRU-ATT model proposed in this paper has better prediction performance than the other three prediction models. This model is helpful for the intelligent regulation of TBM tunneling parameters and provides a more feasible auxiliary intelligent decision-making method for TBM safe and efficient tunneling construction.

\section{Data Availability}

The data used to support the findings of this study are included within the article and can be available from the corresponding author upon request. 


\section{Conflicts of Interest}

The authors declare that there are no conflicts of interest regarding the publication of this paper.

\section{Acknowledgments}

This work was supported by Fundamental Research Funds for the Central Universities (Project No. FRF-TP-20-043A1), Open Research Fund Program of State Key Laboratory of Hydroscience and Engineering (Project No. sklhse-2021-C-04), and Independent Research Project of Huaneng Renewables Corporation Limited (Project No. HNRKJ-2020-04). The costs to publish in open access have been covered by the funding.

\section{References}

[1] Z. Liu, L. Li, X. Fang et al., "Hard-rock tunnel lithology prediction with TBM construction big data using a globalattention-mechanism-based LSTM network," Automation in Construction, vol. 125, no. 3, Article ID 103647, 2021.

[2] J. Li, P. Li, D. Guo, X. Li, and Z. Chen, "Advanced prediction of tunnel boring machine performance based on big data," Geoscience Frontiers, vol. 12, no. 1, pp. 331-338, 2021.

[3] A. Afradi, A. Ebrahimabadi, and T. Hallajian, "Prediction of TBM Penetration Rate Using Fuzzy Logic, Particle Swarm Optimization and Harmony Search Algorithm," Geotechnical and Geological Engineering, vol. 39, 2021.

[4] A. Delisio, J. Zhao, and H. H. Einstein, "Analysis and prediction of TBM performance in blocky rock conditions at the Lötschberg Base Tunnel," Tunnelling and Underground Space Technology, vol. 33, pp. 131-142, 2013.

[5] Q. M. Gong and J. Zhao, "Development of a rock mass characteristics model for TBM penetration rate prediction," International Journal of Rock Mechanics and Mining Sciences, vol. 46, no. 1, pp. 8-18, 2009.

[6] U. Ates, N. Bilgin, and H. Copur, "Estimating torque, thrust and other design parameters of different type TBMs with some criticism to TBMs used in Turkish tunneling projects," Tunnelling and Underground Space Technology, vol. 40, pp. 46-63, 2014.

[7] X. Yang, G. F. Gong, H. Y. Yang, L. H. Jia, and Q. W. Ying, “A cutterhead energy-saving technique for shield tunneling machines based on load characteristic prediction," Journal of Zhejiang University - Science, vol. 16, no. 5, pp. 418-426, 2015.

[8] R. Gertsch, L. Gertsch, and J. Rostami, "Disc cutting tests in Colorado Red Granite: implications for TBM performance prediction," International Journal of Rock Mechanics and Mining Sciences, vol. 44, no. 2, pp. 238-246, 2007.

[9] S. Yagiz, "A model for the prediction of tunnel boring machine performance," Proceedings of 10th IAEG Congress, Article ID 383, pp. 1-10, 2006.

[10] C. Su, "Analysis of mechanical properties of two typical kinds of cutterheads of shield machine," Advanced Science Letters, vol. 4, no. 6-7, pp. 2049-2053, 2011.

[11] Y. Ge, J. Wang, and K. Li, "Prediction of hard rock TBM penetration rate using least square support vector machine," IFAC Proceedings Volumes, vol. 46, no. 13, pp. 347-352, 2013.

[12] H. Krause, Geologische Erfahrungen beim Einsatz von Tunnelvortriebsmaschinen in Baden-Württemberg, Springer, New York, NJ, USA, 1976.

[13] G. Meschke, J. Ninić, J. Stascheit, and A. Alsahly, "Parallelized computational modeling of pile-soil interactions in mechanized tunneling," Engineering Structures, vol. 47, pp. 35-44, 2013.

[14] B. Maidl, M. Herrenknecht, U. Maidl, and G. Wehrmeyer, Mechanised Shield Tunnelling, John Wiley \& Sons, Hoboken, NJ, USA, 2nd edition, 2012.

[15] E. Avunduk and H. Copur, "Empirical modeling for predicting excavation performance of EPB TBM based on soil properties," Tunnelling and Undergroud Space Technology, vol. 71, pp. 340-353, 2018.

[16] Y. Seo, F. J. Macias, P. D. Jakobsen, and A. Bruland, "Influence of subjectivity in geological mapping on the net penetration rate prediction for a hard rock TBM," Rock Mechanics and Rock Engineering, vol. 51, pp. 1599-1613, 2018.

[17] N. R. Barton, TBM Tunnelling in Jointed and Faulted Rock, CRC Press, Boca Raton, FL, USA, 2000.

[18] J. Rostami, Development of a Force Estimation Model for Rock Fragmentation with Disc Cutters through Theoretical Modeling and Physical Measurement of Crushed Zone Pressure, Colorado School of Mines, Golden, CO, USA, 1997.

[19] X. P. Zhou and S. F. Zhai, "Estimation of the cutterhead torque for earth pressure balance TBM under mixed-face conditions," Tunnelling and Underground Space Technology, vol. 74, pp. 217-229, 2018.

[20] Q. Zhang, T. Huang, G. Y. Huang, Z. X. Cai, and Y. L. Kang, "Theoretical model for loads prediction on shield tunneling machine with consideration of soil-rock interbedded ground," Science China Technological Sciences, vol. 56, no. 9, pp. 2259-2267, 2013.

[21] I. Evans, "The force required to cut coal with blunt wedges," International Journal of Rock Mechanics and Mining Sciences \& Geomechanics Abstracts, vol. 2, no. 1, pp. 1-12, 1965.

[22] S. Y. Zhou, Y. L. Kang, C. X. Su, and Q. Zhang, "Study on prediction model of TBM driving total thrust based on mechanical analysis," Journal of Mechanics: Series A, vol. 52, no. 20, pp. 76-82, 2016.

[23] T. Kasper and G. Meschke, "On the influence of face pressure, grouting pressure and TBM design in soft ground tunnelling," Tunnelling and Underground Space Technology, vol. 21, no. 2, pp. 160-171, 2006.

[24] N. Afrasiabi, R. Rafiee, and M. Noroozi, "Investigating the effect of discontinuity geometrical parameters on the TBM performance in hard rock," Tunnelling and Underground Space Technology, vol. 84, pp. 326-333, 2019.

[25] Q. L. Zhang, R. Ma, Y. Hu, Z. Z. An, T. Yin, and Q. B. Li, "Intelligent control theory of thermal stress in mass concrete structures," Journal of Hydroelectric Engineering, vol. 40, no. 5, pp. 11-21, 2021.

[26] A. M. Noori, R. Mikaeil, M. Mokhtarian, S. S. Haghshenas, and M. Foroughi, "Feasibility of intelligent models for prediction of utilization factor of TBM," Geotechnical \& Geological Engineering, vol. 38, pp. 1-19, 2020.

[27] C. Zhou, L. Ding, Y. Zhou, H. Zhang, and M. J. Skibniewski, "Hybrid support vector machine optimization model for prediction of energy consumption of cutter head drives in shield tunneling," Journal of Computing in Civil Engineering, vol. 33, no. 3, Article ID 04019019, 2019.

[28] W. G. Zhang, H. R. Li, C. Z. Wu, Y. Q. Li, Z. Q. Liu, and H. L. Liu, "Soft computing approach for prediction of surface settlement induced by earth pressure balance shield tunneling," Underground Space, vol. 6, no. 4, pp. 353-363, 2021.

[29] W. Sun, M. Shi, C. Zhang, J. Zhao, and X. Song, "Dynamic load prediction of tunnel boring machine (TBM) based on heterogeneous in-situ data," Automation in Construction, vol. 92, no. 5, pp. 23-34, 2018. 
[30] H. Q. Yang, Z. H. Wang, and K. L. Song, "A new hybrid grey wolf optimizer-feature weighted-multiple kernel-support vector regression technique to predict TBM performance," Engineering with Computers, vol. 36, pp. 1-17, 2020.

[31] F. Wang, G. F. Gong, L. W. Duan, and Y. F. Qin, "XGBoost based intelligent determination system design of tunnel boring ma-chine operation parameters," Journal of Zhejiang University, vol. 54, no. 4, pp. 633-641, 2020.

[32] P. Malhotra, L. Vig, G. Shroff, and G. Agarwal, "Long Short Term Memory Network for Anomaly Detection in Time Series," in Proceedings of the 23rd European Symposium on Artificial Neural Networks, Computational Intelligence and Machine Learning, ESANN 2015, Bruges, Belgium, April 2015.

[33] X. Gao, M. Shi, X. Song, C. Zhang, and H. Zhang, "Recurrent neural networks for real-time prediction of TBM operating parameters," Automation in Construction, vol. 15, pp. 130-140, 2019.

[34] S. Hochreiter and J. Schmidhuber, "Long short-term memory," Neural Computation, vol. 9, no. 8, pp. 1735-1780, 1997.

[35] R. Dey and F. M. Salemt, "Gate-variants of Gated Recurrent Unit (GRU) Neural Networks," in Proceedings of the IEEE International Midwest Symposium on Circuits \& Systems, pp. 1597-1600, IEEE, Boston, MA, USA, August 2017.

[36] Y. F. Zhu, W. W. He, J. X. Li, Y. C. Li, and P. Q. Li, "SOC estimation of lithium battery based on Bi-LSTM/Bi-GRU cyclic neural network," Energy storage science and technology, vol. 10, no. 03, pp. 1163-1176, 2021.

[37] P. Xuan, Y. Ye, T. Zhang, L. F. Zhao, and C. Sun, "Convolutional neural network and bidirectional long short-term memory-based method for predicting drug-disease associations," Cells, vol. 8, no. 7, p. 705, 2019.

[38] C. Wang, G. F. Gong, Y. Yang, J. J. Zhou, L. W. Duan, and Y. K. Zhang, "Prediction and analysis of cutter head torque of nsvr hard rock tunnel boring machine," Journal of Zhejiang University: Natural Science Edition, vol. 52, no. 3, p. 479, 2018.

[39] S. K. Hou, Y. R. Liu, and K. Zhang, "Prediction of TBM tunnelling parameters based on IPSO-BP hybrid mode," Chinese Journal of Rock Mechanics and Engineering, vol. 39, no. 8, pp. 1648-1657, 2020.

[40] X. X. Zhou, Q. M. Gong, L. J. Yin, H. Y. Xu, and C. Ban, "Prediction of tunneling parameters in TBM stable section based on BLSTM-AM model," Rock. Mech. Eng, vol. 39, no. S2, pp. 3505-3515, 2020. 